Y. SUN, A. J. DARLING, Y. LI, K. FUJISAWA, C. F. HOLDER, H. LIU, M. J. JANIK, M. TERRONES, R. E. SCHAAK* (THE PENNSYLVANIA STATE UNIVERSITY, UNIVERSITY PARK, USA)

Defect-Mediated Selective Hydrogenation of Nitroarenes on Nanostructured $\mathrm{WS}_{2}$

Chem. Sci. 2019, 10, 10310-10317.

\section{Selective Hydrogenation of Nitroarenes Catalyzed by Nanostructured Tungsten Disulfide}

\section{Category}

Polymer-Supported

Synthesis

\section{Key words}

tungsten catalysis

hydrogenation

nitroarenes

chemoselectivity

anilines

Results:<smiles>Nc1ccccc1</smiles>

$>99 \%$ conversion $>99 \%$ selectivity<smiles>Nc1ccc(C(=O)O)cc1</smiles>

$>99 \%$ conversion $>99 \%$ selectivity<smiles>N#Cc1ccc(N)cc1</smiles>

$>99 \%$ conversion $>99 \%$ selectivity<smiles>C=Cc1cccc(N)c1</smiles>
$94 \%$ selectivity
(<smiles>COC(=O)c1ccc(N)cc1</smiles>

$>99 \%$ conversion $>99 \%$ selectivity<smiles>Nc1ccc(C=O)cc1</smiles>
$78 \%$ selectivity<smiles>CC(=O)c1ccc(F)c(N)c1</smiles>

$>99 \%$ conversion $>99 \%$ selectivity<smiles>NC(=O)c1ccc(N)cc1</smiles>

$>99 \%$ conversion $>99 \%$ selectivity<smiles>Nc1ccc(S(N)(=O)=O)cc1</smiles>

$>99 \%$ conversion $>99 \%$ selectivity<smiles>Nc1ccc(Br)cc1</smiles><smiles>Nc1ccc(I)cc1</smiles>

$>99 \%$ conversion $>99 \%$ selectivity $\left(100^{\circ} \mathrm{C}\right)$<smiles>CCOC(=O)/C=C/c1ccc(N)cc1</smiles>

$>99 \%$ conversion
$>99 \%$ selectivity<smiles>Nc1ccc(Cl)cc1</smiles>

$>99 \%$ conversion $>99 \%$ selectivity
$>99 \%$ conversion $>99 \%$ selectivity<smiles>Cc1ccc(N)cc1</smiles>

$>99 \%$ conversion $>99 \%$ selectivity<smiles>Nc1ccc(Cc2ccncc2)cc1</smiles>

$>99 \%$ conversion $>99 \%$ selectivity
Significance: Nanostructured tungsten disulfide $\left(2 \mathrm{H}-\mathrm{WS}_{2}\right)$ catalyzed the selective hydrogenation of nitroarenes in the presence of other reducible functional groups under $\mathrm{H}_{2}$ (50 bar) to give the corresponding anilines exclusively in $>99 \%$ conversions with 78 to $>99 \%$ selectivities.
Comment: The preparation of $2 \mathrm{H}-\mathrm{WS}_{2}$ was previously reported (B. Mahler, V. Hoepfner, K. Liao, G. A. Ozin J. Am. Chem. Soc. 2014, 136, 14121). In the hydrogenation of 1-nitro-3-vinylbenzene, the catalyst was reused four times without a significant decline in conversion, whereas the selectivity increased during the recycling experiment (fresh: $94 \%$ selectivity; $4^{\text {th }}$ reuse: $>99 \%$ selectivity). 